\title{
Mechatronic Design of a Modular Self-Reconfiguring Robotic System
}

\author{
Cem Ünsal and Pradeep K. Khosla \\ Institute for Complex Engineered Systems \\ Carnegie Mellon University
}

\begin{abstract}
Design and implementation of I-Cubes, a modular selfreconfigurable robotic system, is discussed. I-Cubes is a bipartite collection of individual modules that can be independently controlled. The group consists of active elements, called links, which are 3-DOF manipulators capable of attaching to/detaching from the passive elements (cubes) acting as connectors. The cubes can be oriented and positioned by the links. Using actuation and attachment properties of the links and the cubes, the system can selfreconfigure to adapt to its environment. Tasks such as moving over obstacles, climbing stairs can be performed by changing the relative position and connection of the modules.

The links are actuated using servomotors and worm gear mechanisms. Mechanical encoders and rotary switches provide position feedback for semi-autonomous control of the system. The cubes are equipped with a novel mechanism that provides inter-module attachment. Design and hardware implementation of the system as well as experimental results are presented.
\end{abstract}

\section{Introduction}

Recent research on robotic manipulators includes reconfigurable modular systems for versatility in task orientation, and adaptation to changing environments. An obvious extension of such systems is to combine reconfigurability with autonomous systems to obtain selfreconfiguring systems that can change their shape for adaptation and task-orientation. Our specific aim in designing such a system is to overcome some of the problems encountered by mobile robots, and to design systems that are geared toward multiple tasks. Selfreconfigurable systems emerge as new technologies such as distributed robotic systems and MEMS, move sensing, locomotion and actuation capabilities of the robots further, and toward smaller scales.

We envision a modular self-reconfiguring group that consists of two modules with different characteristics, as detailed in Section 1. A large group of modules that can change its shape according to the locomotion, manipulation, or sensing task at hand, will be capable of transforming into a snakelike robot to travel inside an air duct or tunnel, a legged robot to move on uneven terrain, a climbing robot that can move over obstacles, a flexible manipulator for space applications, or an extending structure to form a bridge.

Designing a modular system with identical elements has several advantages over large and complex robotic systems.
The units can be mass-produced, and their homogeneity can provide faster production at a lower cost. A large system consisting of many elements is less prone to failures, since it would be capable of removing malfunctioning elements from the group and reconfigure its elements. Homogeneous groups of modules that are capable of self-reconfiguring into different shapes also provide a manufacturing solution at the design phase where identical elements are considered, while providing a modular system that can be re-arranged for different tasks in the application phase.

In order to have the advantages listed above, a modular system must have several essential properties, such as geometric, physical and mechanical compatibility among individual modules. Furthermore, several design issues need to be considered for the system to be autonomous. Essential properties of our particular system as well as design issues relating to implementation are given in the following sections.

Previous research on modular robotic systems includes manipulators that can be designed according to task specification [8], modularly synthesized kinematic structures [6], and cellular systems as self-organizing manipulators [1]. These and similar ideas on modularity have been extended to modular structures that can self-reconfigure. Existing 2-D self-reconfiguring systems include Inchworm [3], and selforganizing robots [2] moving in vertical planes, Fractum [12], and metamorphing hexagonal modules [7] moving in horizontal plane. Recent 3-D systems are Polypod that combines different gaits [11], robotic molecule [4] and selfreconfigurable structure [5] that are both capable of changing shape using neighboring elements as pivot points.

The system described in this paper is a modular selfreconfiguring bipartite system that separates the components that provide computation, sensing and power from the components that provide actuation in order to combine different gaits and task-oriented modules with selfreconfiguration capabilities. Next section introduces our approach to self-reconfiguration, defining its characteristics and advantages. Section 3 describes the hardware implementation, while Section 4 presents the experiments conducted with prototypes. Section 5 concludes the paper with a discussion on implementation and future additions.

\section{The Modular Self-Reconfiguring System}

I-Cubes (or ICES-Cubes) are a class of modular selfreconfiguring robotic system. The system is bipartite, i.e., a collection of independently controlled mechatronic manipulators (links) and passive connection elements 
(cubes). Links are capable of connecting to and disconnecting from the faces of the cubes; they can therefore move from one cube to another, or move a cube (Figures 1 \& 2 ). We envision that all links and cubes are capable of permitting power and information flow to attached modules. A group of links and cubes does in fact form a dynamic pseudo-graph [10] where the links are the edges, and the cubes are the nodes. When a link moves, the structure of the graph may change. The system described above has the following properties:

- Modules can be independently controlled (only a cube attached to the non-fixed end of a moving links is affected by link motion).

- All modules have the same characteristics and are mechanically and computationally compatible.

- The 3-D structure fits a cubic lattice to guarantee interlocking of neighboring modules, i.e., the distance $L$ between cubes (while in a position to accept a link) is constant.

- Links have sufficient degrees of freedom to complete motions in 3-D.

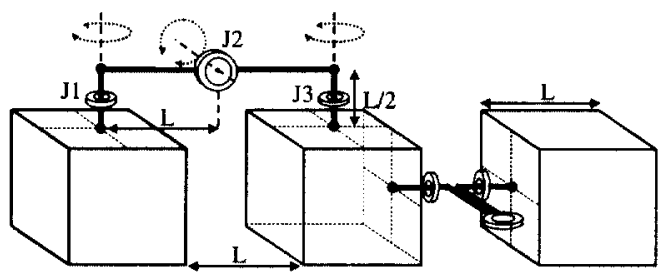

Figure 1. Geometric constraints and joint definitions for the links.

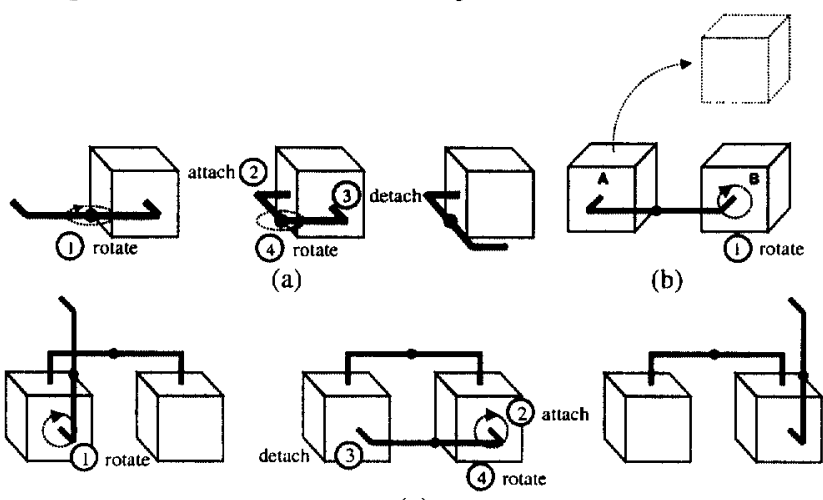

(c)

Figure 2. Examples of link motions.

Since the actuation for self-reconfiguration is provided by the links, cubes can be used to provide computation, sensing and power resources. If the modules are designed to exchange power and information, the cubes may be equipped with batteries, microprocessors and sensors to be the decisionmaking elements while the links become the 'muscles' of the system. It is also possible to remove some of the attachment points on the cubes to replace them with wheeled or threaded systems for faster locomotion. Specifically, we envision small robots that can reposition themselves to form a group that is capable of self-reconfiguring in order to move over obstacles that a single robot cannot overtake. Similar scenarios that combine different gaits with shape reconfiguration include stair climbing and traversing pipes.

\subsection{System design}

As seen in Figure 1, the size definitions for the links and the cubes are dependent. If the length of a cube edge is $L$, then the links should have four sections of length $L / 2$, and $L$. The three rotational degrees of freedom for the links are provided by the joint $\mathrm{J} 2$ at the middle, and the joints located at end segments ( $\mathrm{J} 1$ and $\mathrm{J} 3$ ). Joints $\mathrm{J} 1$ and $\mathrm{J} 3$ are both capable of providing continuous 360 -degree rotations, while $\mathrm{J} 2$ can only rotate 270 degrees. In order to fit the cubic lattice formed by the modules, the links must be able to fully open to provide the required distance $L$ between cubes attached to the link (See the link on the right in Figure 1).

The design parameters given above and the attachment capabilities enable links to (a) move from one cube face to another, (b) move one cube while attached to another, and (c) move from one cube to another (Figure 2). Note that there are other link motions not shown here.

A cube consists of six faceplates with attachment points for link connectors. Cubes do not contribute to the selfreconfiguring motions with the exception of the motion to lock the link connector in place, as described in Section 3.2. A cube attached to a link can be (i) rotated, (ii) translated in vertical or horizontal plane, or (iii) act as a pivot point for a moving link. The role of the cube depends on the position and motion of the active link as well as the connections formed by all modules. For example, same joint rotation may move a link with respect to the cube attached to it or rotate the cube, depending on which end of the link is stationary.

A self-reconfiguring modular system must consist of elements that can attach to and detach from neighboring modules. For the system described here, required attachment mechanism between the links and the cubes need to be mechanically feasible to hold multiple elements together. It must be designed for use with the available degrees of freedom associated with the links. This mechanism must also be energy efficient. A latching mechanism is desirable and actuation for latching should be limited preferably to a few seconds. An ideal candidate is a mechanism that is in locked position when idle, and that can easily switch to unlocked position for a short period during attachment/detachment. The aim is to create a lock that will only cycle between states when actuated, and that needs to be actuated only when changing state for an energy efficient modular robotic system. Section 3 gives the detailed description of the current link and attachment mechanism prototypes.

We have designed our system to overcome some of the common problems in modular self-reconfiguration. Capabilities such as energy-efficient actuation, on-board power resources, information and power transfer between elements and autonomous motion are required for a feasible practical implementation and were considered during system design. Our design of actuators for the links and the connection mechanism significantly increases energy 
efficiency: they need to be actuated only when link or locking motion is required (Section 3.2). The available space inside the cubes can be used for batteries, sensing and control modules for the system. We plan to design electromechanical connectors that will provide power and serial communication. Mechanical encoders and custom-designed positioning switches are used for position feedback (Section $3.1)$.

\subsection{3-D Reconfiguration}

This section illustrates few simple scenarios in which a small group of cubes and links self-reconfigure and move from one position/shape to another. Figure 3 gives snapshots of a possible scenario for a 4L4C group (four links and four cubes). This group is capable of moving to a higher surface by reconfiguring itself (e.g., climbing stairs). Connections between elements are kept such that the system forms a single connected graph at any given time. There are several intervals where multiple links can move simultaneously. Note that cube faces that are initially on the ground and several others are not used during reconfiguration. It may also be possible to find other action sequences that minimize the number of faces used in the reconfiguration process. Black circles on the cubes indicate faces that are initially oriented upward. As seen in the final image, the cubes are still oriented correctly at the end of the action sequence. To guarantee this result, cubes need to be re-oriented in earlier phases of the solution, as seen in images (b) and (c).

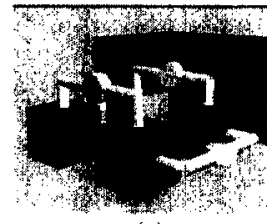

(a)

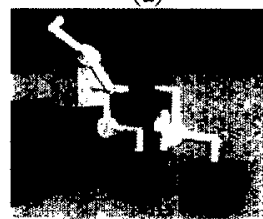

(d)



Figure 3. A 4 L $4 \mathrm{C}$ group self-reconfiguring to move over and obstacle.

Another example that combines a self-reconfiguring system with one that is capable of faster locomotion is shown in Figure 4 . Since the cubes are passive elements that do not contribute to the reconfiguration motion with the exception of locking mechanism, these modules can be equipped with capabilities that provide different gaits (wheels, treads) and task-oriented modules (sensors, cameras, etc.). In Figure 4, the leftmost robot in the first image has a camera, but cannot see what is on/behind the obstacle. These wheeled robots can move into position to form a connected group and selfreconfigure to lift the camera-equipped robot. For an initial configuration and a sequence of actions dependent of the initial configuration, it is possible to move the robot with the camera on top of others. The robot can then detach from the group and continue its surveillance mission. The required number of faces with attachment points is two for the camera-equipped robot. It is also possible for a larger group to self-reconfigure into a tower to lift a surveillance robot to see beyond relatively larger obstacles [9].



(a)

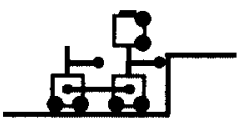

(d)

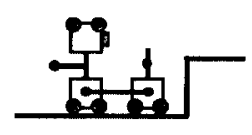

(b)

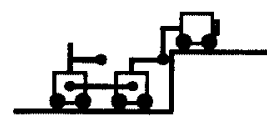

(e)

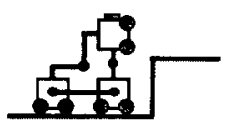

(c)

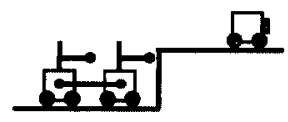

(f)
Figure 4. Two carrier robots lifting another.

The second scenario illustrates an important characteristic of the system. A heterogeneous group of small robots could combine individual robot capabilities with selfreconfiguration to complete a task that would be impossible for individual robots of relatively small size. Some of the robots can carry multiple links in order to enable other teammates to roam around without additional payload (link) while carrying out tasks that require only individual capabilities. Link-carrier robots would provide a 'stepping stone' for other team members.

Also, note that the passive modules do not have to have a cube shape, as long as their attachment points fit the cubicle lattice illustrated in Figure 1.

\section{Implementation}

This section describes the hardware implementation of the links, the attachment mechanism, and the feedback control for link and cube motions.

\subsection{The links}

The links have three worm gear mechanisms driven by small servos to provide continuous rotation at the end joints, and 270-degree rotation at the middle joint (Figure 5). Using custom plastic pieces, the servos are coupled to the worms. At the end joints, the wheels are aligned with the crossshaped connectors that attach to cube faces. At the middle joint, the servo and the worm are located on one side of the body, while the wheel and its shaft are attached to the other side. Thus, rotating the servos will rotate the connector shafts at the ends, or move one side of the link with respect to the other at the middle. The normal distance from one wheel shaft to another is again equal to $L$. The potentiometer feedback circuitry and mechanical stops on servos are removed to obtain continuous 360 -degree turns on the worm shaft. 
There are two main advantages of using a worm gear coupled with the servos. First, the torque provided by the servo is multiplied by the ratio of the gear mechanism (1:40). Second, the system is an energy efficient solution for actuation. Since, the wheel cannot drive the worm, servos do not have to be actuated all the time to hold the links in a specific position.

Mechanical encoders coupled with the worms at both ends of the link provide 25 counts per revolution. With 1:40 gear ratio, each pulse corresponds to 0.36 degrees turn of the end joint.

We have designed a rotary switch with four contact points coupled with the middle wheel shaft. A connection point fixed to the link body part that includes the worm is used to generate pulses at each 90 -degree turn. This mechanism can be extended for higher resolution feedback signal using multiple contact points. We plan to increase the number of contact points for better resolution.
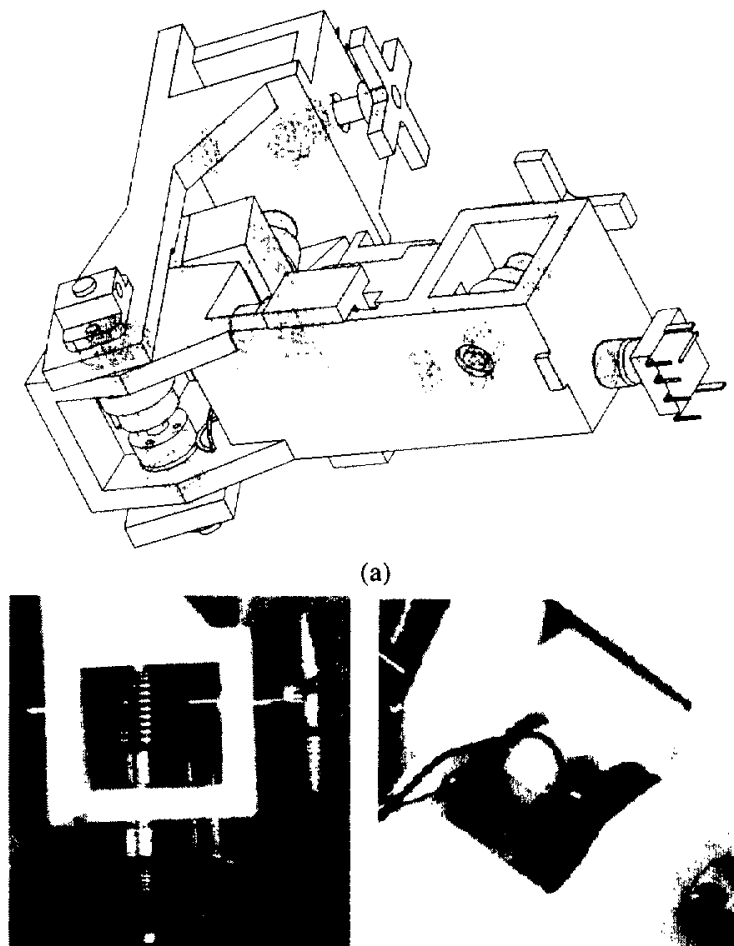

(b)

Figure 5. The link: (a) CAD image of the link assembly, (b) details of the latest prototype (Also see Section 4).

The link body is implemented using prototyping plastic shaped by Genisys $^{\circ}$ fused deposition modeling (FDM) machine. Files for link and cube bodies, generated by ProEngineer ${ }^{\circ} \mathrm{CAD}$ program, are sent to this 3-D printer for creating complete pieces that require minimal assembly with off the shelf components, which are modeled in ProEngineer. All components are assembled with custom designed parts to guarantee compatibility and unrestricted motion of the modules. Mechanical components such as worms, wheels, bearings, are available commercially. Size limitations on these off the shelf components impose a constraint on the size of the prototype. A link equipped with servos, wormwheel pairs, complete with all other mechanical and electrical components weights approximately $370 \mathrm{gr}$. The length $L$ is $8 \mathrm{~cm}$. One side of the link body is indented slightly toward the connector to enable the link to complete 270-degree turns around its middle joint. All shafts are keyed to couple with the plastic servo heads and encoder shafts. The servos are held in place with custom-designed plastic holders.

\subsection{The attachment mechanism and the cubes}

The design of the attachment mechanism between the crossshaped link connectors and the cube faces is based on the requirements listed in Section 2.1 and the mechanical capabilities of the links. The mechanism is useful in a situation described here, where the connecting elements need to have a single point of contact and a rotational degree-offreedom with respect to each other.

A cross-shaped link connector is designed to enter (2) and rotate (3) to its 'locked' position inside an opening (receptor) on the cube faceplate (Figure 6). This inhibits lateral motion separating the link and the cube. Before the connector's approach, sliding pegs under the surface of the faceplate retract (1) to clear the path of the connector. Once the connector is in 'locked' position, the sliding pegs return (4) to their extended (idle) position. This action inhibits the free rotation of the connector, thus locks the link and the faceplate together. In this position, a joint motion will rotate one of the modules (link or cube) with respect to the other.

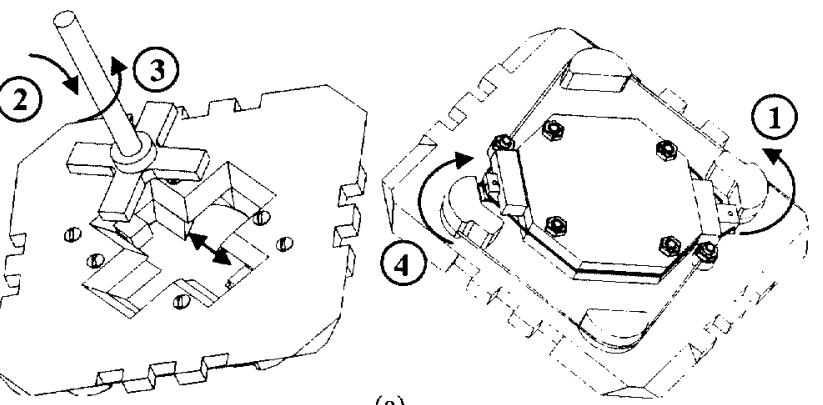

(a)

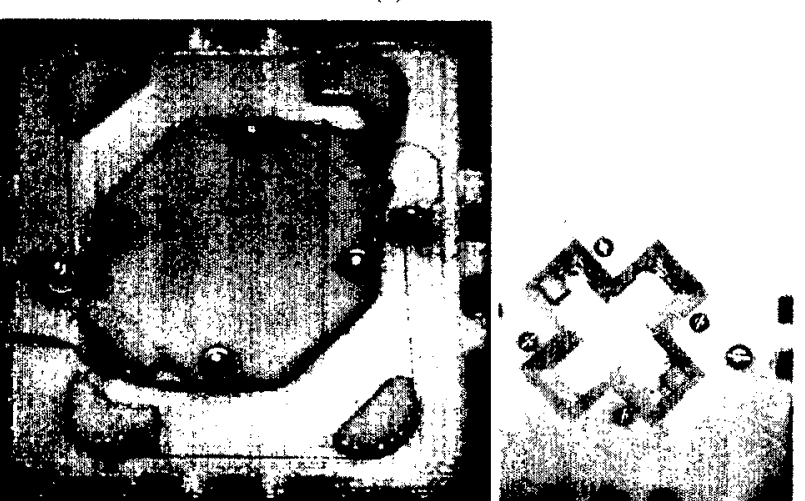

(b)

Figure 6. Cross-shaped connector with twist-and-lock mechanism: (a) CAD images, and (b) the prototype. 
The sliding pegs are actuated by a shape memory alloy wire (Nitinol) of diameter $100 \mu \mathrm{m}$ and a rubber band or spring. A rubber band pulls (or a spring push) the pegs into their idle position. The SMA wire attached to the sliding pegs retracts almost instantaneously when current is applied to it, and the pegs slide outward to clear the connectors path. The pegs can be hold in position by a continuous current, and will return to their idle position in less than $0.5 \mathrm{sec}$ after the current is cut. Since the retraction ratio for Nitinol is approximately $4 \%$, the wire is wrapped around plastic guides to obtain necessary length for the required motion ( $10.5 \mathrm{~cm}$ in Figure 6).

The edges of the receptor are chamfered to guarantee that the connector clears the cube face when approaching the locking position. Note that the connector does not approach the receptor from the normal, but following a curve that is normal to the faceplate only at the surface of the supporting layer under the faceplate. The edges of the connector are rounded to facilitate sliding into locked position. Note that the cross-shaped connector must be correctly oriented with respect to the opening on the faceplate for successful attachment. In order to detach the link from a cube face, the actions indicated in Figure 6 should be carried out in reverse order.

We have designed individual faceplates with male/female matching edges. This enables us to use the faceplates on a platform to test links, or combine them to form cubes with different number and type of attachment mechanisms (See Section 4 for images). One faceplate for the cube weights approximately $20 \mathrm{gr}$. The depth of a faceplate is $1 \mathrm{~cm}$; therefore, when six faceplates are combined to form a cube, the maximum available volume inside the cube for computational elements, sensor and power source is slightly larger than the volume of a $6-\mathrm{cm}$ cube. Previous designs for the attachment mechanism are discussed in $[9,10]$.

\subsection{Link and Cube Control}

The actuators on the links and the cubes are controlled by microprocessors connected to a GUI on a PC via RS-232 serial connection (Figure 7). The links and the cubes can also be controlled manually using simple multi-button controllers. The controller for the attachment mechanisms on the faceplates simply carries out the 'open/close' commands received via the serial link or the 3-button controller. Six output lines drive the transistor switches turning the current on and off on two 15 SMA wires that are connected in series. $5 \mathrm{~V}$ supply is provided by the voltage regulator on the microcontroller circuitry.

Link control circuitry includes a $\mathrm{HCll}$ microprocessor, mechanical encoders, one custom-designed rotary switch, and three servos. The servos are controlled by $20 \mathrm{~ms}$ pulse width modulation (PWM) signal. The $25 \mathrm{cpr}$ encoders coupled to the worm gear provide the feedback pulses that are counted for comparison with the desired number of pulses (i.e., degree rotations). Each pulse corresponds to 0.36 degrees of rotation of the connector. The rotary switch coupled with the middle worm wheel has four contact points.
Thus, pulses from the rotary switch correspond to 90-degree turns of the wheel. The controller adjusts the speed of the servo using proportional control with gain scheduling.

Two $6 \mathrm{~V} 650 \mathrm{mAh}$ rechargeable batteries are used to power all servos on the links, and the attachment mechanisms on the cubes as well as the microprocessors driving the actuators. We are currently updating our Java graphical user interface (GUI) to provide more information about the effects of a single 90-degree action over the group of links and cubes before actually carrying out the action.

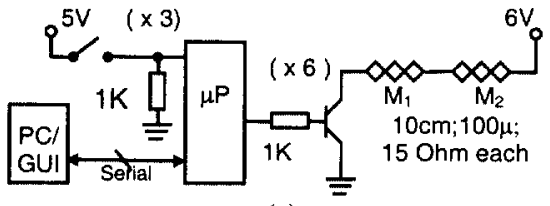

(a)



(b)

Figure 7. Controllers for (a) faceplate and (b) link actuation.

\section{Experiments with Prototypes}

The prototypes described in Section 3 are used to test the feasibility of the system. Tests show that a link can transfer from a horizontal plane to vertical plane, and vice versa. As shown in Figure 8, a link can translate itself between two horizontal connection points; it then attaches its free end to the vertical faceplate, and detaching the other end from the horizontal plate, it is capable of moving in vertical plane. Similarly, a link can move from one cube face to another as well as from one cube to another (Figure 9) using the three available degrees of freedom.

The links are also capable of moving and exchanging cubes. Figure 10 shows two links and 5-faced cube. Link \#1 (left) moves the cube into position and orients it for link \#2 to attach (Figure 10a-b). After link \#2 connects to the cube, link \#1 detaches. Link \#2 is now free to move the cube to its next destination (Figure 10c-d). Similar examples for different link and cube configurations are given in $[9,10]$.

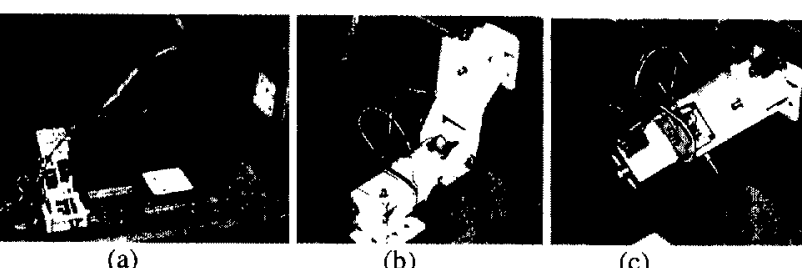

Figure 8. A link moving from horizontal plane to vertical plane. 




(a)

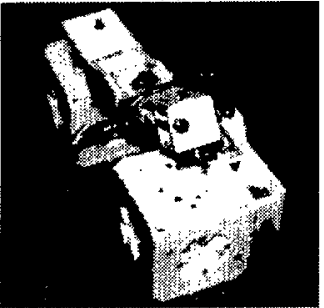

(c)

Figure 9. A link transferring from one cube to another.



(a)

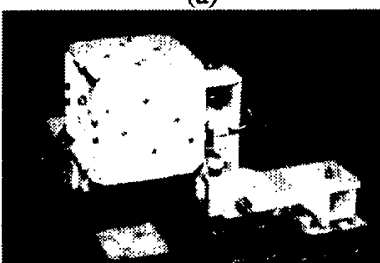

(c)

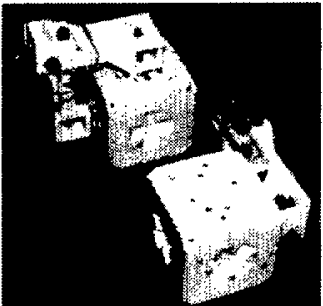

(b)

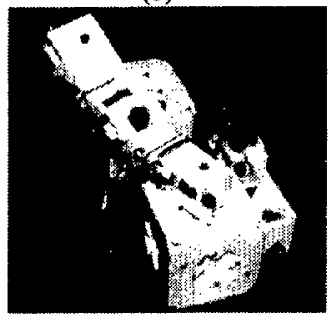

(d)



(b)

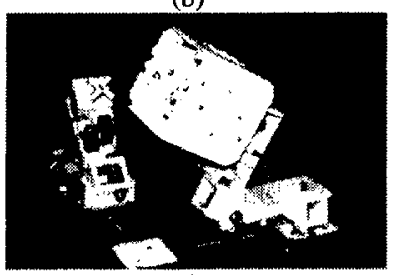

(d)
Figure 10. Two links lifting, moving and exchanging a cube.

\section{Conclusions and Discussion}

We have presented a 3-D modular robotic system that can self-reconfigure to adapt to its environment. Design requirements for energy efficiency, feasibility and a taskoriented robotic system are given. Implementation of the active and passive modules based on these requirements is discussed. A novel attachment mechanism designed for modularity in the reconfigurable system and motion control method for the active elements are presented. Several scenarios illustrating the capabilities and advantages of the system and hardware experiments with current prototypes are also given.

Although it complicates the system design and implementation, partitioning the system into two different modules combines the ability to change shape with different and faster types of statically stable locomotion modes, and present a possibility for sensor-equipped, self-powered modules that can be geared toward a specific task. Furthermore, the design approach described here enables us to reduce the minimum number of elements for stable motion, while providing a feasible system that may be autonomous and energy-efficient.
Our plans include updating the microcontrollers for intermodule communications and smaller size. Simple electromechanical connectors that can provide multiple lines for power and serial communication lines between modules are also considered. The surface of the cross-shaped attachment piece will include the contact points. Using flexible cable wrapped around the connector shaft while forcing counter rotations to unwrap the cable when a link end is free and design of contact rings enabling continuous rotations are the two alternatives we consider. The GUI will also be updated to provide more information about the actuation for manual control and will be interfaced to a motion planner [10]. We are currently designing rotary switches for 45-degree resolution, and new links and cubes to reduce the module size $L$ to $6 \mathrm{~cm}$.

\section{Acknowledgements}

This work is supported by DARPA and the Institute for Complex Engineered Systems. The authors would like to thank William F. Hein, Ryan J. Thomas, and Stephen M. Wolfe for their valuable contribution on design, implementation, and testing of I-Cubes.

\section{References}

[1] Fukuda, T., and Y. Kawauchi, "Cellular Robotic System as One of the Realization of Self-Organizing Intelligent Universal Manipulator," Proc. IEEE Conf. on Rob. \& Auto., pp. 662-667, 1990.

[2] Hokasawa, K., et al, "Mechanisms for self-organizing robots which reconfigure in a vertical plane," Distributed Auto. Rob. Sys. 3, Springer-Verlag, 1998, pp. 111-118.

[3] Kotay, K., and D. Rus, "Self-reconfigurable Robots for Navigation and Manipulation," Proc. of Intl. Symp. on Exp. Robots, 1997.

[4] Kotay, K., D. Rus, M. Vona, and C. McGray, "The Selfreconfiguring Molecule: Design and Control Algorithms," Algorithmic Foundations of Robotics, A.K. Peters, 1998.

[5] Murata, S., H. Kurokawa, E. Yoshida, K. Tomita, and S, Kokaji, "A 3-D Self-Reconfigurable Structure," Proc. IEEE Intl. Conf. on Rob. \& Auto., pp. 432-439, 1998.

[6] Neville, B., and A. Sanderson, "Tetrabot Family Tree: Modular Synthesis of Kinematic Structures for Parallel Robotics," Proc. IEEE/RSJ Intl. Symp. of Rob. Res., pp. 382-390, 1996.

[7] Pamecha, A., C.-J. Chiang, D.Stein, and G. Chiricjian, "Design and Implementation of Metamorphic Robots," Proc. ASME Design Eng. Tech. Conf. \& Comp. in Engineering Conf., Irvine, CA, 1996. [8] Paredis, C.J.J., and P.K. Khosla, "Kinematic Design of Serial Link Manipulators from Task Specifications," Intl. Jour. of Robotics Research, Vol. 12, no. 3, pp. 274-287,1993.

[9] Ünsal, C., H. Kılıçcöte, and P. K. Khosla, "I(CES)-Cubes: A Modular Self-Reconfigurable Bipartite Robotic System," Proc. of SPIE Sensor Fusion and Decenralised Ctrl. in Rob. Sys. II, vol. 3839, pp. 258-269, Boston, MA, 1999.

[10] Ünsal, C., H. Kılıç̧̧̋te, and P. K. Khosla, "A Modular Selfreconfigurable Bipartite Robotic System: Implementation and Motion Planning," submitted to Auto. Robots Journal.

[11] Yim, M., "New locomotion Gaits," Proc. IEEE Intl. Conference on Robotics and Automation, San Diego, CA, 1994.

[12] Yoshida, E., et al, "Experiments of Self-Repairing Modular Machine," Distributed Auto. Rob. Sys.3, Springer-Verlag, 1998, pp. 119-128. 